\title{
ROYAL SOCIETY MEDAL AWARDS, 1947
}

$\mathrm{T}$ HE following is the substance of the remarks made by Sir Robert Robinson, president of the Royal Society, in presenting the medal awards for 1947 at the anniversary meeting on December 1 .

\section{Copley Medal}

The Copley Medal is awarded to Emeritus Prof. G. H. Hardy in recognition of his distinguished contributions to mathematics [died December 1].

For many years G. H. Hardy has occupied an outstanding position among mathematicians in Great Britain, while his eminent services and original work have also been very widely recognized in other countries. In his memoirs and papers published with collaborators, especially J. E. Littlewood, are to be found the origins of the major developments of 'classical analysis' throughout the world in the years 1910-35.

Among the most influential and original of his works are those dealing with the Tauberian theorem for Cesáro summability (1910); the proof that the Riemann zeta-function has an infinity of zeros on the critical line (1914); the asymptotic formula for the number of partitions of an integer (with Ramanujan, 1918) ; the papers on Waring's problem and Goldbach's problem (with Littlewood, 1920, 1922); the paper in Acta Mathematica on Fourier series (with Littlewood, 1930). He has made important contributions to lattice-point theory, to the theory of integral equations, and (with Littlewood) to the theory of Diophantine approximation.

Lying in widely separated domains of analysis, all these papers contain fundamentally novel ideas which were the starting-points for new significant developments by Hardy and others. For example, a part of the distinguished work of Vinogradoff may justly be said to derive from that of Hardy. His influence has been exerted, not only by his publication of original work, but also through a long succession of pupils, many of whom are now well-known mathematicians. At a time when the vigorous treatment of analysis in university courses had still to win general acceptance, Hardy's enthusiastic advocacy and his inspiring lectures had a decisive effect. In addition, the appearance of his highly individual text-book "Pure Mathematics", helped to demolish finally the barriers between the British and Continental schools of mathematics.

It is impossible to characterize in a few sentences the achievement, so varied, and so abstruse, of the Copley Medallist for 1947, but in very ordinary words it may be said that Hardy saw clearly what were the most important tasks and problems lying before mathematicians; he had good judgment in selecting those which seemed ripe for attack, and he displayed the greatest skill and energy in their solution.

\section{Royal Medals}

A Royal Medal is awarded to Prof. C. N. Hinshelwood, for his outstanding researches in chemical kinetics.

Prof. Hinshelwood has brought a characteristic lucidity of mind and directness of approach to bear on the investigation of many classical problems presented by the course of chemical change. He has clarified these subjects to an extent which seems remarkable to those who recall the mystery which enshrouded them only a few years ago.

The acute suggestion of Lord Cherwell relating to the consequences flowing from the relative rates of formation and decomposition of an intermediate additive complex was taken up and extended by Hinshelwood. He predicted that on the basis of this theory certain apparently monomolecular reactions should follow a bimolecular law at low pressures. One example of the justification of this prophecy was found in the decomposition of acetaldehyde. This experimental verification of the hypothesis of intermediate complexes of definite life, and the new light shed on the mechanism of activation of molecules, are pillars of modern chemical kinetics. Hinshelwood has enlarged our knowledge of this subject by many other researches of great importance including those on the synthesis of water, a classical problem of seeming simplicity and real complexity, and those on the conditions of 'chain' reactions. His ingenious experimental methods, though characterized by a lack of unnecessary elaborations, have nevertheless always been able to provide accurate results.

It was a very original conception to apply the principles and methods of chemical kinetics to the growth of bacteria, and Hinshelwood has already made noteworthy progress in this field. The outcome has been to provide a new and more delicate assessment of the presence and activity of factors that influence growth at all stages under defined conditions.

During the War he carried out important researches on the adsorption of gases by charcoal, and on the preparation of suitable adsorbents for different purposes, ranging from gas-masks to the production of penicillin.

The brilliant exposition of chemical topics contained in Prof. Hinshelwood's books places contemporary chemists and students still further in his debt. His memorable addresses on the wider implications of chemistry made as president of the Chemical Society and on the occasion of the celebration of its centenary were generally acclaimed. Possessing enviable linguistic attainments, he has frequently been an 'ambassador of British science'. Recognizing these achievements we wish to honour Prof. Hinshelwood above all for his pioneering work in the laboratory and in the study, which has thrown so much light on the most fundamental processes of chemistry, the mode of interaction of molecules.

A Royal Medal is awarded to Dr. F. M. Burnet for his distinguished contributions to our knowledge of the viruses.

Dr. Burnet, director of the Walter and Eliza Hall Institute, at Melbourne, has made a series of extremely significant investigations of bacteriophages and viruses since 1925. In twenty-seven papers on the first topic he has made greater advances than anyone apart from d'Herelle. He has elucidated the phenomenon of lysogenesis or 'earrying' of a bacteriophage, active against other bacteria, by an organism which is itself apparently unaffected. He has shown how study, partly on a biochemical basis, of the range of activity of 'phages against various bacteria 
can be used to classify not only the 'phages but also the bacteria themselves. Other investigations have made an important contribution to our present ideas as to what bacteriophages are.

Since 1933 he has studied many viruses pathogenic for vertebrates. He has shown how psittacosis is endemic in wild Australian parrots, and has thrown light on the epidemiology of herpes and poliomyelitis. To modern workers he is best known for his development of several techniques for cultivating viruses in living chick embryos, and their application in immunological and other studies of viruses, especially influenza. He has written monographs on the use of chick embryos in virus research, on immunity to viruses, on influenza and on the nature of antibodies.

At present he is examining the agglutination of mammalian and avian red blood cells by a number of viruses and virus-products, and is thus throwing fresh light on the reactions taking place between viruses and the surfaces of susceptible cells. He has found that an enzyme produced by cholera vibrios has an action on blood cells which is quite similar to that of influenza viruses, and is capable of blocking the union between virus and susceptible cell. This discovery indicates one line of approach to the problem of the chemotherapy or chemoprophylaxis of virus infections.

Burnet has been active in many other fields; he has studied staphylococcal toxins and he was the first to make a formolized staphylococcal toxoid. $\mathrm{He}$ has demonstrated that a rickettsia is the cause of Australian $Q$-fever.

While his experimental studies have demanded close attention to detail, certain continuous threads have connected his interests. The breadth of his outlook and his philosophic attitude of mind are well exemplified in his fascinating book "Biological Aspects of Infectious Diseases" and in the more recent publication "Viruses as Organisms".

\section{Davy Medal}

The Davy Medal is awarded to Prof. Linus Pauling in recognition of his distinguished researches on molecular structure.

The unusual breadth of Prof. Pauling's scientific knowledge, his mastery of physics and mathematics in addition to ohemistry, made him peculiarly fitted to apply quantum mechanics to problems of chemical valency bonds. In him, theoretical acumen and penetrating insight are associated with experimental skill of the highest order, and thus his theories were often tested after the devising of now physical techniques. This combination of theoretical and practical attainments is very remarkable at such a level in both aspects.

As a natural consequence of his catholic interests, Pauling's work has covered a very wide range of chemical structural problems. He has devoted much attention to line spectra and many other physical topics; but his most individual achievements are in the advance of molecular structure theory. In elucidating the fundamental structure of simple organic compounds he developed the method of localized pairs (atomic orbitals) to provide a theoretical treatment of the valency conditions of such molecules. By this introduction of orbital hybridization he gave the first theoretical account of the tetrahedral distribution of the valencies of the saturated carbon atom (1928). This was followed by the introduction of the quantum mechanical concept that the actual state of a molecule such as benzene should be represented as a resonance hybrid of several valency bond structures (1931). The application of this idea and the experimental verification of its structural implications in terms of increased stability and decreased bond distances greatly increased our understanding of aromatic and general chemistry. The calculus of the resonance theory of Pauling was the first to introduce a quantitative treatment of valency problems, and its effect has been felt far beyond the bounds of the experimental material.

In 1939 his extensive work was summarized in a great book "The Nature of the Chemical Bond", which will always be one of the classics of chemistry. Here we find a general classification of stubborn aspects of valency theory along with valuable tables of generalized and correlated data on resonance energy terms, bond distances and angles, and atomic radii.

Proceeding to apply his methods to the study of more complex molecular systems, Pauling has made important contributions to our understanding of the metallic bond; of the structure and mode of denaturation of native proteins; and of the mechanism of the fundamental reactions of immunology, that is, antibody formation, and the antibody-antigen interaction.

Prof. Pauling has earned our gratitude, not only for his personal services, great as those have been, but also because he has been a focus of activity and a constant source of inspiration to other workers.

\section{Buchanan Medal}

The Buchanan Medal is awarded to Sir Edward Mellanby for his services to medical science and in recognition of his outstanding researches on dietary factors.

The Buchanan Medal is awarded every five years in respect of distinguished services to the science or practice of hygiene in the direction either of original research, or of professional, administrative, or constructive work, without limit of sex or nationality. In each of these raspects the award to Sir Edward Mellanby is surely one of the most appropriate that could be made. For some thirteen years he has de. voted himself to the service of medical science and the national organisation of medical research. His personal research work, which has been continued right up to the present time, has been concerned mainly with problems of nutrition, and has had important results in the relation of diet to the public health.

Sir Edward's two most important contributions have been on the effects of deficiency of vitamin $D$ and of vitamin $\mathrm{A}$ in the diet. He first showed that rickets was unquestionably due to the lack of a fat-soluble vitamin, since known as vitamin $D$, and he also demonstrated that other factors, dietary and general, such as lack of exercise, contributed to aggravate the condition. Quite recently he has shown that lack of vitamin $A$, in addition to the already recognized effects on general health, is capable of producing profound neurological disorders, owing to the mechanical effect of abnormal growth of bones around the foramina through which nerves leave the central nervous system. The knowledge which has been made available as a result of his investigations has played an important part in the feeding of the nation on scientific lines, in war, and in peace-time.

The study which he made of the effects of lack of iodine has thrown much light on the various forms 
of goitre, and especially on the hyperplastic and colloid forms : the former he showed to be caused by iodine deficiency, particularly in young animals, or in ante-natal states, while the latter is produced by the belated admission of iodine to such sufferers from the hyperplastic condition. The knowledge has been of value in connexion with the lack of iodine in the water and soil of some districts.

Mellanby's researches on the physiological effects of alcohol have also provided valuable knowledge concerning the effects of this substance as a constituent of the diet.

His most recent contribution has been to show that bread which is made from flour bleached by the 'Agene' process, that is, with nitrogen trichloride, can cause the condition in dogs known as canine hysteria.

As the secretary of the Medical Research Council, Sir Edward has proved himself a wise, strong and withal a helpful administrator.

\section{Hughes Medal}

The Hughes Medal is awarded to Prof. J. F. Joliot in recognition of his outstanding researches in nuclear chemistry and physics.

Prof. Joliot, director of the Nuclear Chemistry Laboratory of the Collège de France, was early concerned with investigations leading to the discovery of the neutron and with subsequent work on that particle. In 1932 he found that the very penetrating radiation emitted when beryllium is bombarded with alpha-particles was able to eject protons from hydrogenous material upon which it fell. This observation was followed up by Chadwick, who was able to show that the radiation consisted of the neutrons which he and Rutherford had sought for years.

In 1934 Joliot made a discovery of the utmost importance. He found that aluminium and other light-atom substances were rendered radioactive by bombarding them with alpha-particles. He showed that these radioactive products emitted positive or negative electrons and gamma-rays. By chemical methods he proved that the radioactivity produced was due to the formation of unstable isotopes of neighbouring elements; for example, in the case of aluminium, the active substance produced was an isotope of phosphorus. It was soon shown that the production of these artificially radioactive isotopes was possible by bombardment with other types of particle and that practically all the elements could be prepared in radioactive form. In this way Joliot founded an important development of modern 'tracer' technique and opened up a field of investigation of immense importance to physical, chemical, and biological science.

In 1939 Joliot and his co-workers observed that in the process of fission of uranium several neutrons are emitted. This important observation indicated the possibility of a chain process in uranium, and Joliot at once endeavoured to produce such a self-propagating reaction. With Halban and Kowarski he showed, in 1940, that if the fission neutrons were slowed down in heavy water a chain-reacting system could be produced. The practical success of the heavy-water 'piles' in the United States of America and in Canada is the outcome of these pioneering discoveries in the application of atomic energy.

In a great part of his work, Prof. Joliot has been closely associated with his wife, Prof. Irène JoliotCurie.

\section{SYNTHESIS OF PHOSPHOLIPIDS IN THE FETTUS}

\author{
By G. POPJÁK
}

Beit Memorial Fellow for Medical Research, St. Thomas's Hospital Medical School, London

$\mathrm{W}$ HETHER fœtal lipids are derived primarily from the preformed maternal substances by placental transmission, or from synthesis within the fœetus, cannot yet be answered satisfactorily. Up to now, most investigators have been concerned with the placental transmission of fatty (and other) sub. stances, and very little has been done to explore the possibility of synthesis of these compounds by the fotus. Nielson ${ }^{1}$ studied the permeability of the rat placenta to phospholipids with the aid of $\mathrm{P}^{32}$ and found that after the injection of the mother with inorganic phosphate, labelled with $\mathrm{P}^{32}$, radioactive phospholipids could be isolated from the fœtuses within $2 \mathrm{hr}$. after the injection. When, on the other hand, an emulsion of phospholipids labelled with $\mathrm{P}^{32}$ was injected intravenously to the mother, the appearance of radioactive phospholipids in the foetuses was very much delayed, and even then only small amounts were found. Nielson, therefore, concluded that the placenta does not transmit phospholipids and that these substances are readily synthesized within the fœtus. In my experience, when an emulsion of phospholipids is injected intravenously, so long as any of the excess phospholipid remains in the circulation, it always stays in the form of an emulsion. The only conclusion, therefore, that can be drawn from Nielson's work is that the placenta does not transmit emulsions of phospholipids; whether this is also true for the plasma phospholipids in the physiological state of dispersion remains an open question. In order to follow the experimental design of Nielson in the investigation of the sources of foetal phospholipids, the mothers should be transfused with plasma containing labelled phospholipids. Up to now, the supplies of $\mathrm{P}^{32}$ in Great Britain have been small, and therefore this experiment, requiring relatively large amounts of the isotope, is not practicable.

There is, however, another possible way of attacking this problem. After the injection of a suitable dose of $\mathrm{P}^{32}$ in an inorganic form, the plasma phospholipids become labelled with this isotope. If fotal phospho. lipids are derived from the preformed maternal substances only, then the radioactivity of fotal phospholipids should never exceed the activity of the maternal plasma phospholipids. If, on the other hand, a rapid synthesis takes place within the fotus, and if the transfer of inorganic phosphate from the mother to the foetus is sufficiently rapid, then there might conceivably be a time when the activity of the foetal phospholipids is greater than the activity of the maternal plasma phospholipids.

Pregnant rats, guinea pigs and rabbits were injected subcutaneously or intravenously with disodium hydrogen phosphate labelled with $\mathrm{P}^{32}$. After varying intervals, the phospholipids were isolated from the maternal plasma, maternal liver, fœtal liver, fœtal carcass and viscera, fœtal placenta, and whenever possible from the fotal plasma also, and their radioactivities compared. In several experiments, the inorganic phosphate of the tissues was precipitated as the ammonium-magnesium salt from a trichloracetic acid extract and its radioactivity measured 